\title{
Impact of Vessel Wall MR Imaging in the Work-Up for Ischemic Stroke
}

I nterest in the diagnostic utility of intracranial vessel wall MR imaging (VW-MR imaging) has increased during the past 2 decades. Many studies have shown that VW-MR imaging provides supplemental anatomic information to existing lumenbased imaging techniques. ${ }^{1,2}$ Yet, the diagnostic utility of VWMR imaging in the work-up of ischemic stroke still remains investigative, and questions remain about whether it provides diagnostic information that ultimately improves patient outcomes.

In a comprehensive study design, the authors take the first step in addressing this question. In this single-center retrospective case series from 2006 to 2014, the authors measure the impact of VW-MR imaging to see whether supplementing the conventional diagnostic work-up with inclusion of multicontrast VW-MR imaging changed the stroke neurologist's working diagnosis in 205 cases. Stroke etiologies were classified using a modified version of the Trial of ORG 10172 in Acute Stroke Treatment $(\text { TOAST) })^{3}$ classification scheme by a stroke neurologist before and after incorporating information from VW-MR imaging reviewed by an experienced neuroradiologist. The authors report that VW-MR imaging frequently altered the etiologic classification. In particular, a higher proportion of cases were attributed to intracranial atherosclerotic disease and fewer cases were categorized as "intracranial arteriopathy, not otherwise specified," "etiology undetermined due to 2 or more potential causes," and "small vessel occlusion." On the basis of these results, the authors conclude that VW-MR imaging has substantial impact in the work-up of stroke etiologies.

The results are interesting for several reasons: First, the study attempts to measure the value of VW-MR imaging by assessing the frequency in the change of the working diagnosis of stroke etiologies if additional vessel wall imaging information is made available to the stroke neurologist. At the authors' institution under the direction of expert panels, a measurable impact was detected. It would be most interesting to know whether in a prospective design, if a change in therapeutic management would follow, indicating confidence in the diagnostic interpretation or in a retrospective study including diagnostic follow-up information to assess diagnostic accuracy. In a slightly different study design, Kesav et $\mathrm{al}^{4}$ also assessed the potential impact of VWMR imaging by measuring the reclassification of stroke etiologies with the addition of VW-MR imaging. Kesav et al also suggest that VW-MR imaging impacts the diagnostic evaluation with diagnostic etiology reclassification in cases originally classified as "undetermined" etiologies and large (intracranial) artery atherosclerosis.

This leads to a second interesting aspect of the study. The study results suggest that intracranial atherosclerosis could be a more common ischemic stroke etiology than previously thought. Among the 205 cases, 116 were reclassified as "intracranial atherosclerosis" following VW-MR imaging. One possible explanation may be the ability of VW-MR imaging to detect prestenotic or angiographically occult atherosclerotic lesions. ${ }^{5-7}$ The detection of such culprit lesions may, in part, decrease the expensive downstream work-up costs of cryptogenic stroke.

Third, in the analysis, the time interval between symptom onset and VW-MR imaging was not significantly associated with a change in etiologic classification. This could be explained by the possibility of the studied sample comprising largely of chronic or long-standing diseases such as atherosclerosis as opposed to more acute vasculopathies such as reversible cerebral vasoconstriction syndrome. Moreover, in cases such as artery-to-artery embolism within the intracranial arterial vasculature, timing may be key to detecting enhancing culprit lesions with plaque surface irregularity. ${ }^{8}$ As a future direction, it would be informative to examine the importance of the timing of VW-MR imaging to address its impact in acute primary treatment decisions, which, in turn, would also address secondary prevention.

Finally, the results of this study give rise to optimism, given the improved techniques of VW-MR imaging since 2006 and could potentially be more impactful than reported. For instance, VW-MR imaging using 3D techniques with whole-brain coverage suggest improved assessment of vasculitis as well as intracranial atherosclerosis. ${ }^{9,10}$

As the field of stroke continues to advance, intracranial VWMR imaging has diagnostic potential but, as the authors cautiously acknowledge, remains an area of active research. Next, it would be most informative to show diagnostic accuracy, which 
could be achieved by incorporating data from clinical and imaging follow-up to assess clinical improvement. Such an analysis would yield results that would help persuade one that VW-MR imaging provides diagnostic information that may ultimately improve patient outcomes.

\section{REFERENCES}

1. Song JW, Obusez EC, Raymond SB, et al. Vessel wall MRI added to MR angiography in the evaluation of suspected vasculopathies. $J$ Neuroimaging 2019;29:454-57 CrossRef Medline

2. Mossa-Basha M, Shibata DK, Hallam DK, et al. Added value of vessel wall magnetic resonance imaging for differentiation of nonocclusive intracranial vasculopathies. Stroke 2017;48:3026-33 CrossRef Medline

3. Adams HP, Jr, Bendixen BH, Kappelle LJ, et al. Classification of subtype of acute ischemic stroke. Definitions for use in a multicenter clinical trial. TOAST. Trial of Org 10172 in Acute Stroke Treatment. Stroke 1993;24:35-41

4. Kesav P, Krishnavadana B, Kesavadas C, et al. Utility of intracranial high-resolution vessel wall magnetic resonance imaging in differentiating intracranial vasculopathic diseases causing ischemic stroke. Neuroradiology 2019;61:389-96 CrossRef Medline

5. Song JW, Rafla SD, Obusez EC, et al. High-resolution vessel wall MR imaging in prestenotic intracranial atherosclerotic disease. $\mathrm{J} \mathrm{Clin}$ Neurosci 2019;63:278-80 CrossRef Medline
6. Chung J, Kim BJ, Sohn $\mathrm{CH}$, et al. Branch atheromatous plaque: a major cause of lacunar infarction (high-resolution MRI study). Cerebrovasc Dis Extra 2012;2:36-44 CrossRef Medline

7. Klein IF, Lavallée PC, Mazighi M, et al. Basilar artery atherosclerotic plaques in paramedian and lacunar pontine infarctions: a high-resolution MRI study. Stroke 2010;41:1405-09 CrossRef Medline

8. Wu F, Song H, Ma Q, et al. Hyperintense plaque on intracranial vessel wall magnetic resonance imaging as a predictor of arteryto-artery embolic infarction. Stroke 2018;49:905-11 CrossRef Medline

9. Eiden S, Beck C, Venhoff $\mathrm{N}$, et al. High-resolution contrastenhanced vessel wall imaging in patients with suspected cerebral vasculitis: prospective comparison of whole-brain 3D T1 SPACE versus 2D T1 black blood MRI at 3 Tesla. PLoS One 2019;14: e0213514 CrossRef Medline

10. Wu F, Ma Q, Song H, et al. Differential features of culprit intracranial atherosclerotic lesions: a whole-brain vessel wall imaging study in patients with acute ischemic stroke. J Am Heart Assoc 2018;7 CrossRef Medline

(1) J.W. Song Department of Radiology University of Pennsylvania

http://dx.doi.org/10.3174/ajnr.A6241
Philadelphia, Pennsylvania 
$\mathrm{n}$ the article commentary "Impact of Vessel Wall MR Imaging in the Work-Up for Ischemic Stroke" (Song JW. AJNR Am J Neuroradiol 2019;40:1707-08. 10.3174/ajnr.A6241), the author omitted a citation for the article discussed. The referenced article is "Diagnostic Impact of Intracranial Vessel Wall MRI in 205 Patients with Ischemic Stroke or TIA" (Schaafsma JD, Rawal S, Coutinho JM, et al. AJNR Am J Neuroradiol 2019;40:1701-06. 10.3174/ajnr.A6202).

http://dx.doi.org/10.3174/ajnr.A6353 